\title{
Formative Study on the Wearability and Usability of a Large-Volume Patch Injector
}

\author{
Jakob Lange (iD) \\ Andreas Schneider ${ }^{\prime}$ \\ Christoph Jordi ${ }^{1}$ \\ Michael Lau ${ }^{2}$ \\ Timothy Disher ${ }^{2}$ \\ 'Ypsomed Delivery Systems, Ypsomed \\ AG, Burgdorf, Switzerland; ' 2 Insight \\ Product Development, Chicago, IL, USA
}

Background: The subcutaneous self-administration of biologics using a single large-volume bolus dose requires novel large-volume patch injectors. However, the usability and wearability of such on-body devices has rarely been investigated thus far. Therefore, this formative simulated use experiment studies the overall handling and acceptability in terms of the size and weight of a novel $10 \mathrm{~mL}$ large-volume patch injector device platform.

Methods: Twenty-three participants, including patients and healthcare professionals, simulated two injections with the large-volume patch injector, each lasting $17 \mathrm{~min}$. During the injections, the patient participants performed predefined movements and activities with the on-body devices. Perceived usability and wearability were assessed through observation by the moderator and participant-reported feedback using five-point Likert scales and openended interviews.

Results: All participants successfully completed the simulated injections. Only non-serious usability issues were identified. Users rated the device acceptability in terms of wearability and usability with high ratings.

Conclusion: The results suggest the safe and effective usage of a novel prefilled largevolume patch injector that enables the subcutaneous delivery of a single bolus dose of up to $10 \mathrm{~mL}$ with an injection duration of $15 \mathrm{~min}$. The participants of the simulated use study successfully used the device regardless of the disease state, age, or body size and habitus.

Keywords: self-medication, subcutaneous injection, large-volume injection, bolus dose, drug delivery, usability, medical devices

\section{Introduction}

The subcutaneous delivery of therapeutics continues to drive change in global healthcare systems as it shifts the point of care from hospitals to homes. ${ }^{1}$ Subcutaneous treatment options not only reduce total costs and simplify access to therapy but also improve patient compliance and convenience. ${ }^{2-5}$ Simple-to-use drug delivery devices are instrumental for the safe and effective self-administration of therapeutics for chronic indications, such as psoriasis, arthritis, or multiple sclerosis. ${ }^{6-10}$ The selfinjection of a single volume of up to $2 \mathrm{~mL}$ with prefilled hand-held autoinjectors has been studied extensively, and the recent market uptake of related products provides empirical evidence of their commercial viability. ${ }^{11-13}$ Recently, scholars have explored the feasibility of single large-volume subcutaneous injections with a capacity greater than $2 \mathrm{~mL} .{ }^{14}$ Advances in formulation technologies have facilitated effective dispersion of larger single-dose volumes in the subcutaneous tissue ${ }^{15}$ and have thereby enabled less frequent drug self-administration to positively impact quality of life, treatment adherence, and therapy outcomes. ${ }^{16}$ However, conventional
Correspondence: Jakob Lange Ypsomed AG, Brunnmattstrasse 06, Burgdorf, 340I, Switzerland Tel +4l 344243187

Email jakob.lange@ypsomed.com 
hand-held autoinjectors may be inappropriate for the subcutaneous delivery of larger single-dose volumes with longer injection duration. ${ }^{13}$ Therefore, large-volume patch injectors have emerged as a device category for the safe and effective self-administration of single bolus doses with a capacity greater than $2 \mathrm{~mL}{ }^{17}$ This novel device category of large-volume patch injectors should be distinguished from wearable infusion pumps for administering therapeutics such as insulin or pegfilgrastim using pre-programmable or personalized continuous infusion profiles. ${ }^{18,19}$ To date, the first large-volume patch injector that has been marketed can deliver a single dose of $3.5 \mathrm{~mL}$ of $420 \mathrm{mg}$ of evolocumab every month. ${ }^{20}$ Several large-volume patch injector platforms are under development, ${ }^{17,21}$ and the results of a clinical study on the functional performance, injection site tissue effects, drug depot location, subject pain tolerance, and device acceptability of a new $5 \mathrm{~mL}$ platform were recently reported. $^{22}$

However, although the pharmacokinetics, tolerability, and safety of subcutaneously delivering large single-dose volumes has received considerable scientific attention, ${ }^{11,23-28}$ there are limited studies on the usability and patient perceptions of large-volume patch injector technologies that enable safe and effective selfadministration. As Collins et $\mathrm{al}^{17}$ succinctly summarized, "[u]ser preference studies for large volume, on-body selfinjection systems are needed to better understand usability and inform large volume device designs." If device usability drives preferences and treatment choices of patients and healthcare professionals (HCPs), then the question of how they operate and perceive the usage experience of said devices is worth exploring.

This work examines the feasibility and user perceptions of self-injections using a large-volume patch injector that delivers a single-dose volume of up to $10 \mathrm{~mL}$ from a cartridge-based primary container. Specifically, data on usability (ie, overall handling) and wearability (ie, acceptability of size and weight) were collected from simulated self-injections. During a non-interventional observational study, 23 participants, including patients and HCPs, simulated the use of a large-volume patch injector, performed predefined movement and activity tasks, and reported their experience in terms of device usage, comfort while moving, and confidence while wearing the device.

\section{Methods}

Usability and wearability data were collected from an observational simulated use study based on single-site visits. The study complied with the principles of the Usability Professionals' Association Code of Professional Conduct for Usability Practitioners ${ }^{29}$ and the European Pharmaceutical Market Research Association Code of Conduct, $^{30}$ as well as with the principles outlined in the declaration of Helsinki. The study protocol, informed consent form, and relevant supporting information were reviewed and approved by an institutional review board, Advarra IRB (Pro00037054), prior to study initiation. Participants, and parents or legal guardians for participants under the age of 18, provided informed consent, and also consented to the publication of anonymized responses from the study.

\section{Materials}

Two injections were simulated for each participant using a prototype without needle of a large-volume patch injector device (YpsoDose $^{\circledR}$, Ypsomed AG, Burgdorf, Switzerland), as illustrated in Figure 1.

The device is an on-body, single-dose, and prefilled injector suitable for administering up to $10 \mathrm{~mL}$ of liquid drug with an injection duration of up to $30 \mathrm{~min}$. The device is intended for subcutaneous injections and is placed on the abdomen or thigh. It is held in place by a medical-grade adhesive. A single button activates the device after placement on the skin, with a sensor that determines when it is in contact with the skin. The device automatically inserts the needle when activated. It contains a light-emitting diode-based progress bar and status lights, as well as a red warning light and audible indicator to indicate an error state. The injection progress is visible through a drug window on the device (movement of the plunger). The devices used in the study were fully functional, including the lights, sounds, and sensors; however, they did not contain a needle or liquid filling. The prototype devices were remotely monitored and controlled with a wirelessly connected laptop. The user steps required to simulate an injection with the study devices are shown in Figure 2. The devices used in the study had dimensions of $125 \mathrm{~mm} \times 59 \mathrm{~mm} \times 27 \mathrm{~mm}$ and a weight of $133 \mathrm{~g}$.

An instruction for use was also presented to participants. This six-page document included an overview of device features, a step-by-step operation procedure, and a description of error states.

\section{Participants}

Twenty-three subjects from Chicago, IL, USA, were recruited for this study. Patients (group G1, $n=17$ ) and 


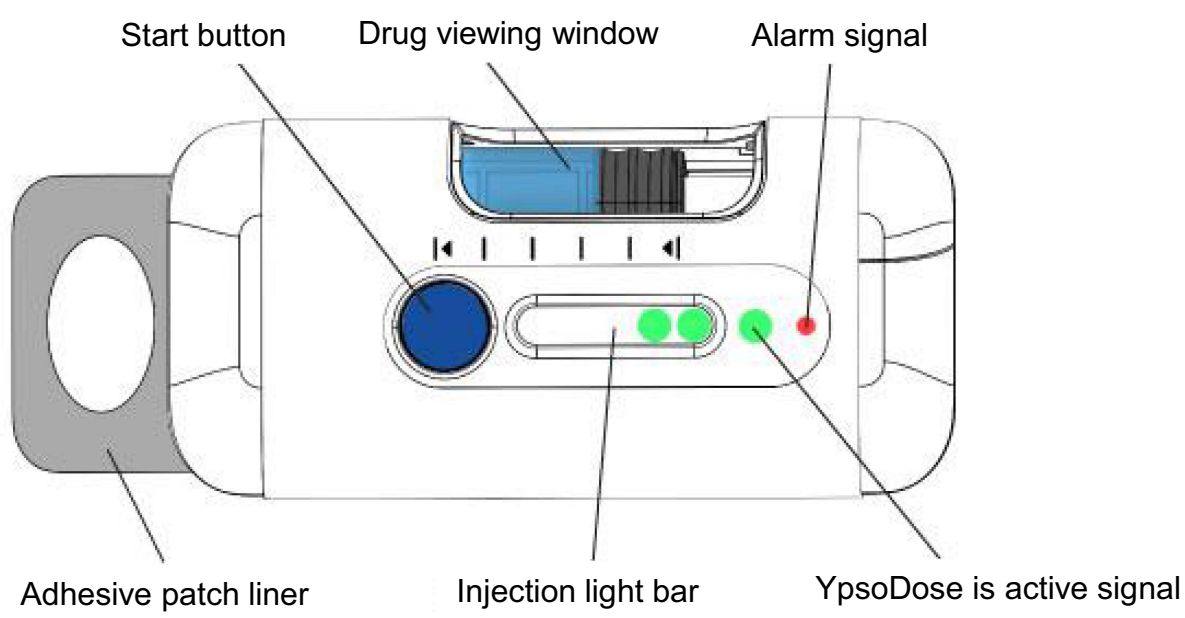

Figure I Large-volume patch injection device used in the simulated use study.

HCPs (group G2, n =6) were included to reflect the intended usage population. Of the 16 female and 7 male participants, 21 had previous experience with injections. The patients in group G1 were diagnosed with at least one of the following diseases, which are amenable to treatment by self-injection: cancer, multiple sclerosis, rheumatoid arthritis, psoriasis, Crohn's disease, asthma, or cardiovascular disease.

The HCPs (G2) were experienced with inpatient (n $=5)$ and outpatient $(\mathrm{n}=1)$ care, and their experience was related to on-body injections and infusions $(n=3)$ or syringe and autoinjector injections $(\mathrm{n}=3)$. Table 1 lists the demographics, disease states, and injection-related experience of the participants. The participants received a small financial compensation after the study.

\section{Study Design}

The study consisted of single in-person, one-on-one simulated use sessions lasting between 60 and $75 \mathrm{~min}$. The oneon-one sessions comprised four primary activities: 1) introduction of the study purpose and device and collection of participant background, 2) device training, 3) simulated use, and 4) discussion, filling out of questionnaires, and concluding the session. The study activities are shown in Figure 3.

Participants were presented with an overview of the study purpose. All participants (G1 and G2) were asked about their experience with injection devices and the infusion process; moreover, the HCPs and patients were asked to describe their current procedures when administering or receiving infusions or injections, respectively. The moderator trained the participants on the operation of the large- volume patch injector. This included an overview of the purpose of the device, a description of its features, and a rehearsal of the usage instructions. Additionally, the moderator described the indication of an error state and the appropriate response in the case of an error.

Participants utilized two devices for two different use scenarios during the study. The scenarios varied between patient (G1) and HCP (G2) participants. For G1 participants, in the first scenario, they initially selected the injection site (abdomen or thigh) and simulated a complete selfadministration process using the device. The target injection duration was preset for $17 \mathrm{~min}$. During the injection process, the patients performed several movement activities, such as standing up, bending to "tie their shoe", and walking around in a circle. In addition, participants had a task to pack or unpack groceries, which involved lifting and bending over. The moderator recorded any user difficulties or errors. During the second scenario, the device was triggered to indicate an error at a certain point after the movement-based activities. The patients attached the device to an alternative location (thigh or abdomen) that was not used during the first scenario. In the case of HCPs (G2), the two scenarios differed from those for the patients as the device in both cases was attached to an injection pad and no movement-related tasks were completed.

As part of the simulated use study, a survey questionnaire was administered to all participants. Items were developed to assess various aspects related to usability and wearability of the patch injector. Both patient and HCP participants were required to complete the patch injector ease-of-use questionnaire. Patients (G1) were also tasked with completing a wearability-related questionnaire. The items in the 


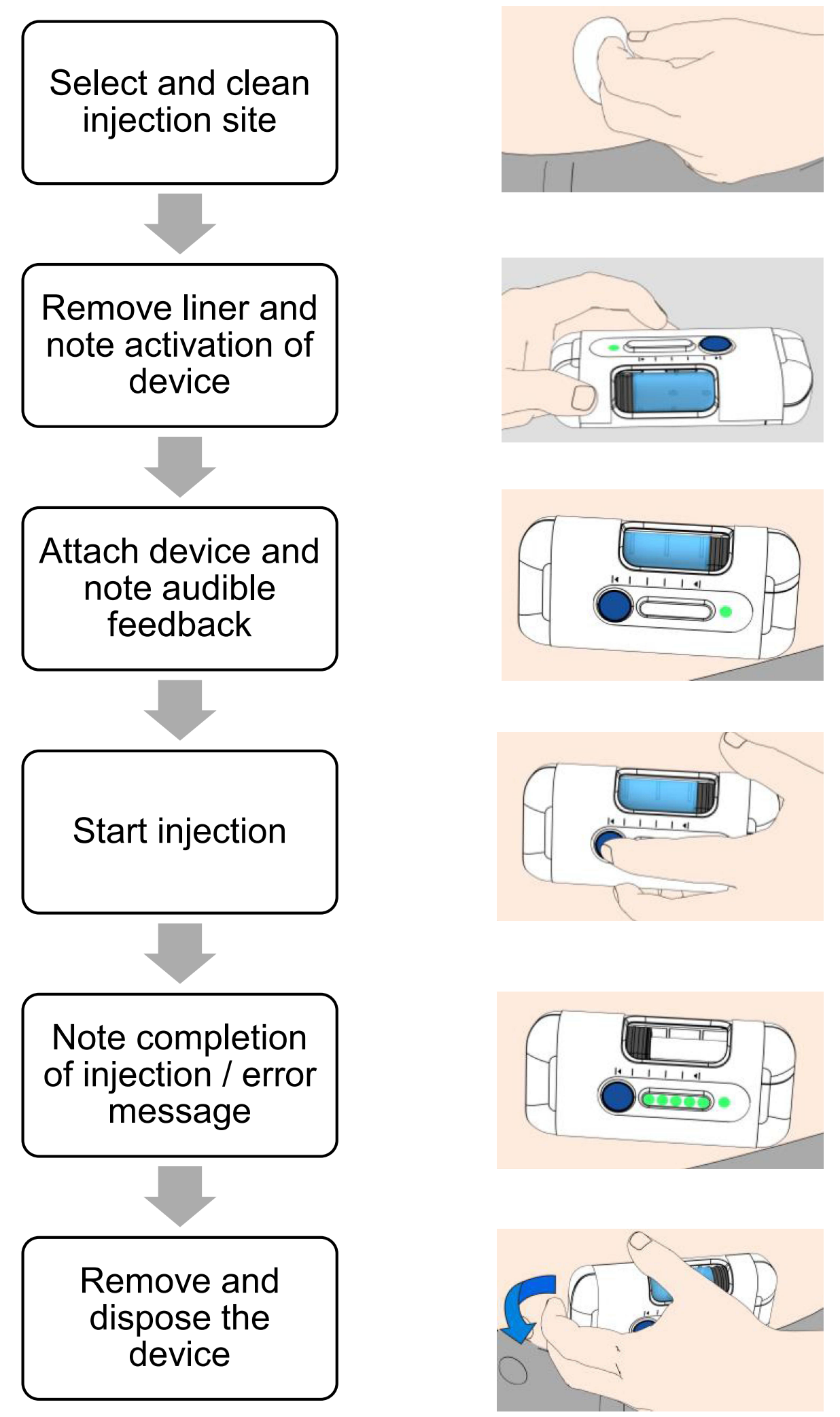

Figure 2 User steps required to perform a simulated injection with the large-volume patch injection device. 
Table I User Population and Disease States in the Simulated Use Study (A and B)

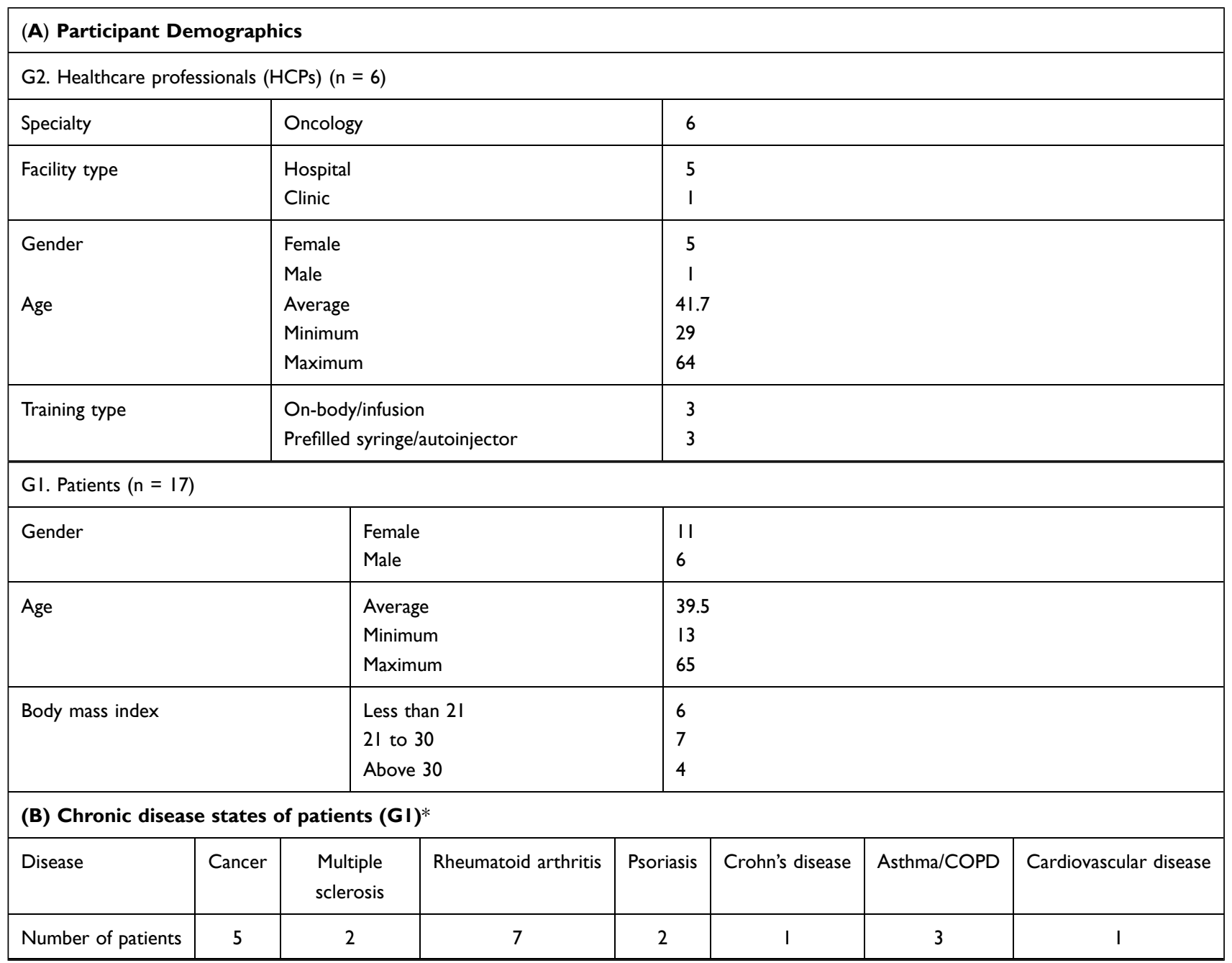

Note: *Certain patients suffered from more than one chronic condition.

questionnaires were designed based on a review of relevant literature and the use steps of the large-volume patch injector included in the study. Each item was measured on a fivepoint Likert scale $(1=$ strongly disagree to $5=$ strongly agree). Descriptive statistical analyses were performed using the statistical software STATA version 12 (StataCorp, College Station, TX, USA).

\section{Results}

\section{First Impressions}

Participants responded favorably to the patch injector prototype, citing an expected advantage in terms of convenience over current standard of care. Multiple participants recognized the value addition when using a large-volume patch injector at home for injection therapies, which would otherwise be administered in a clinical or hospital setting.
This observation was particularly pronounced for oncology patients who welcomed an alternative to their current complex treatment process and considerable time commitments in relation with inpatient care and infusion centers.

Certain participants expressed an initial concern with the size, weight, and security of attachment of the patch injector prototype. The most prevalent concern was the size of the device. Participants perceived it to be large and therefore difficult to attach and maintain a secure attachment during use. Selected statements that illustrate the first impression of the participants when they were shown the device are listed in Table 2.

\section{Usability}

In the first simulated injection, participants were generally successful at achieving a complete simulation of drug delivery using the device. Across both the patient and 


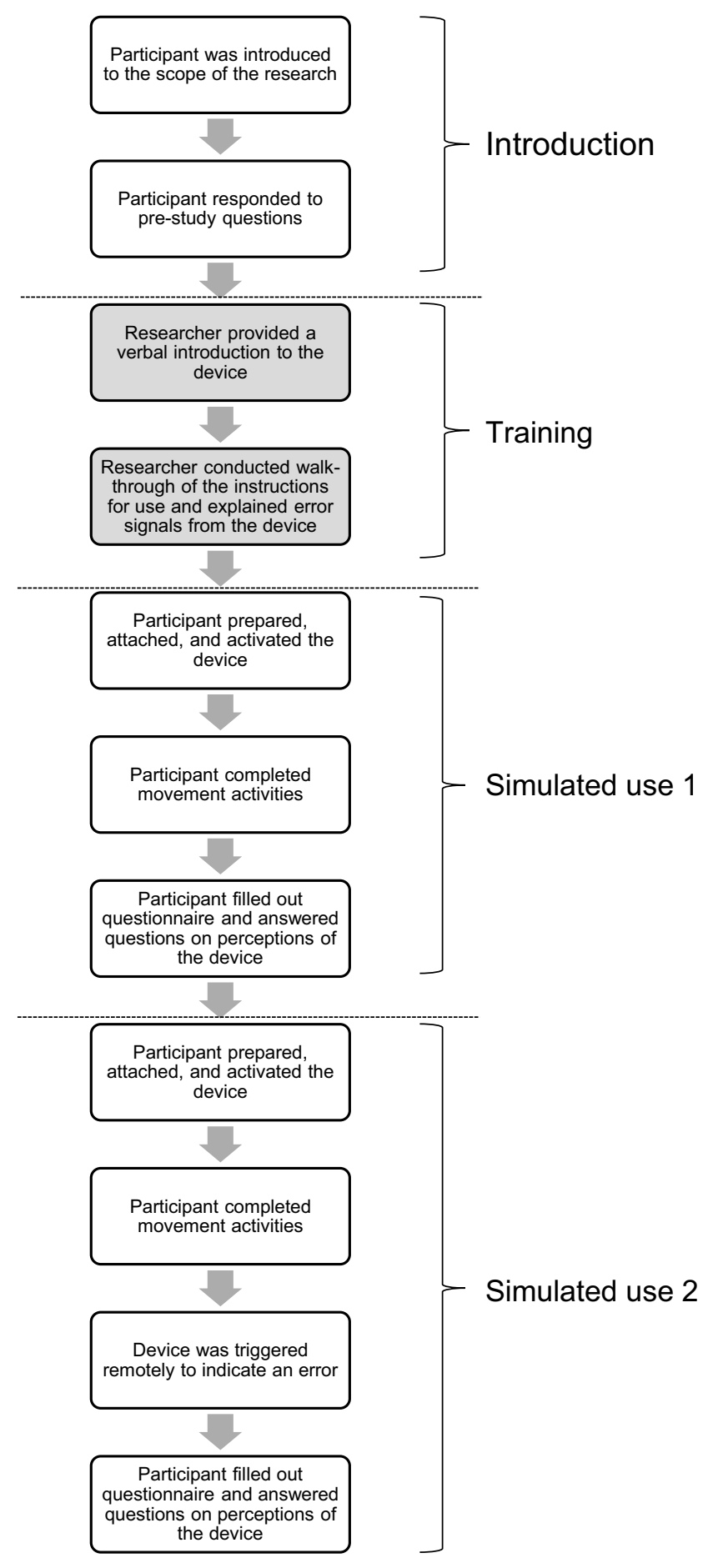

Figure 3 Procedure involved in the non-interventional simulated use study based on single-site visits.

HCP groups, only a single participant in the patient group (G1) experienced an unexpected usability challenge. This participant had excessive body hair and, in his first trial, attempted to stick the device over the hair. When it became obvious that it would not adhere to his skin, he removed and relocated the device. At this point, the
Table 2 Statements Illustrating the First Impression of the Participants When Encountering the Large-Volume Patch for Self-Injection

\begin{tabular}{|c|c|}
\hline Statement & Comment \\
\hline $\begin{array}{l}\text { "If it's going to relieve me from } \\
\text { seeing a doctor then there's } \\
\text { a benefit. The needle's got to be } \\
\text { really small so it shouldn't be too } \\
\text { painful." }\end{array}$ & $\begin{array}{l}\text { Participant suffering from } \\
\text { multiple sclerosis, who is } \\
\text { currently on intravenous } \\
\text { treatment }\end{array}$ \\
\hline $\begin{array}{l}\text { "It goes directly on your body, } \\
\text { cool. I like that a lot." }\end{array}$ & $\begin{array}{l}\text { Participant suffering from } \\
\text { rheumatoid arthritis, who is } \\
\text { currently using autoinjector for } \\
\text { treatment }\end{array}$ \\
\hline $\begin{array}{l}\text { "The only thing l'm concerned } \\
\text { with is if it's going to stay on." }\end{array}$ & $\begin{array}{l}\text { Participant suffering from } \\
\text { multiple sclerosis, who is } \\
\text { currently on intravenous } \\
\text { treatment }\end{array}$ \\
\hline $\begin{array}{l}\text { "Like I said, it's kinda bulky. But } \\
\text { I know it has to be this size } \\
\text { because of the vial." }\end{array}$ & $\begin{array}{l}\text { Participant suffering from } \\
\text { multiple sclerosis, who is } \\
\text { currently treated with vial-based } \\
\text { injections in a clinical setting }\end{array}$ \\
\hline $\begin{array}{l}\text { “It's definitely heavier, a lot more } \\
\text { medication than I'm pretty sure } \\
\text { I was seeing. Honestly with that } \\
\text { device you didn't see the } \\
\text { medicine at all. It didn't have } \\
\text { a button to start it, it would just } \\
\text { start at a specific time." }\end{array}$ & $\begin{array}{l}\text { Patient suffering from cancer, } \\
\text { treated with intravenous } \\
\text { injections and a patch injector, } \\
\text { while comparing the YpsoDose } \\
\text { to the } 0.6 \mathrm{~mL} \text { Neulasta device } \\
\text { she is using }\end{array}$ \\
\hline
\end{tabular}

adhesive was compromised, and the device subsequently came off his body. All 23 participants were successful in the second trial in operating the device appropriately up to the point where the preset error state was triggered. The overall injection success rates for each injection for all participants are illustrated in Figure 4.

In addition to the overall success, all participants were successful in completing individual user steps. Regardless of their body size and shape, participants were able to find sufficient space to place the device and generally found it to be comfortable and secure to wear. All user errors and difficulties observed during the simulated injections are listed in Table 3. In general, only few user errors and difficulties were observed. The only issues observed for more than one participant were not cleaning the injection site prior to attachment (two participants, both during the second injection), there were certain difficulties in activating the device after attachment (four participants, 


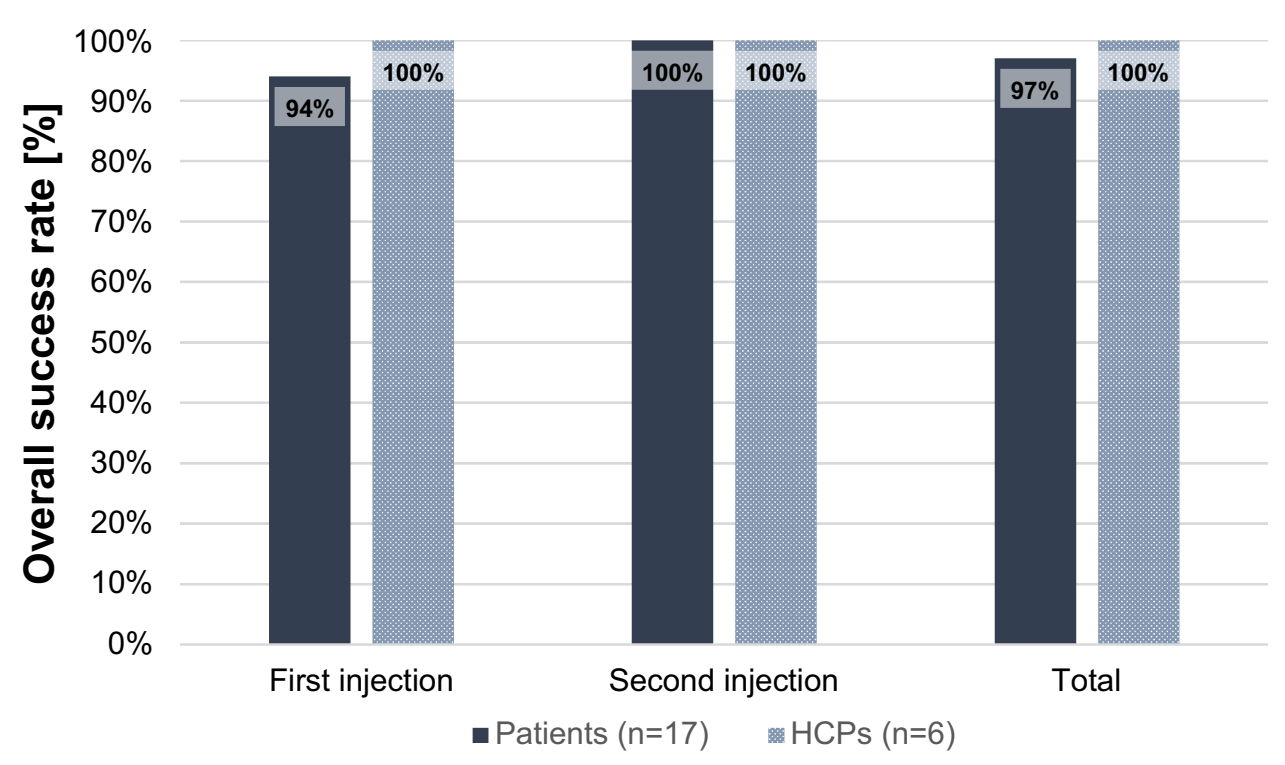

Figure 4 Injection success rates per participant group for first injection, second injection, and overall. An injection was rated to be successful if the user was able to successfully complete a complete simulated injection, regardless of usage errors or difficulties.

all during the first injection), and proper disposal of the device in the sharps disposal container after completion was not performed in certain cases (two participants, both during the second injection).

Participants were able to interpret the training and instructions for usage guidance to select an appropriate initial injection site. All participants cleaned the site for the first injection; however, two participants omitted this step for the second injection. Participants were also able to remove the adhesive liner. Participants heard the audible feedbacks and were able to follow the instructions to verify if the device was activated. No participant had an issue in interpreting the initial activation sounds or light. In all cases, the participants were able to locate an appropriate injection site, orient the device, and securely attach it to the skin, although one small-sized participant initially struggled to identify a suitable and sufficiently large space to place the device on the abdomen. There was some confusion about device status caused by the reminder beeps that were emitted by the device prior to commencement of the drug delivery, but no user errors were observed. Specifically, four participants were unsure about when to press the button to activate the device. Nevertheless, all participants succeeded in activating the large-volume patch injector as intended.

During the simulated injections, participants were able to clearly identify that the injection process was proceeding and verify the progress and status of dose delivery. All participants were able to determine the state of the patch injector by checking the visual progress indicators, ie, the visual feedback lights and plunger movement in the drug window. Most participants did not have any issue detecting the occurrence of an error state. Only one participant in the patient group did not note the change in status of the device when the error state was activated. When prompted by the moderator, the hearing-impaired participant said he did not notice the alarm.

Participants were able to remove the device without difficulty and knew that it had to be discarded in the sharps bin. All participants successfully completed the task during the first injection. Two participants did not dispose the device in a sharps disposal container after the second simulated injection. In both cases, the participant appeared to be using the device to point out and discuss unrelated topics with the moderator, indicating that this error most likely is a study artifact.

Overall, patient participants (G1) made more errors and had more difficulties than HCPs (G2), with the user error and difficulty rate for patients being almost twice as high as the rates for HCPs. The error rate decreased marginally between the first and second injection in G1 but was constant in G2. There was no difference in the type of errors and difficulties between the two participant groups.

After each simulated injection, participants were asked to complete a questionnaire with feedback on the usability of the device. The results are presented in Figure 5 and Table 4. Participants predominantly assigned high rating for the ease of use of the large- 


\begin{tabular}{|c|c|c|c|c|c|c|c|c|c|c|c|c|c|c|}
\hline & & & 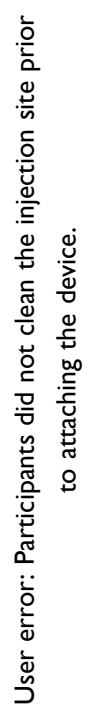 & & 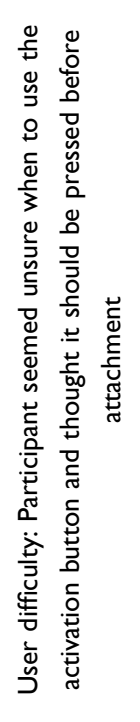 & 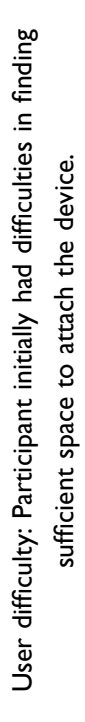 & & 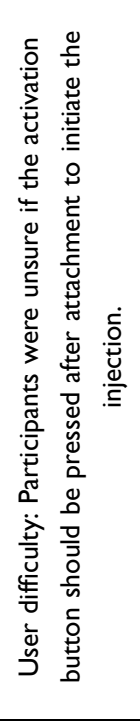 & 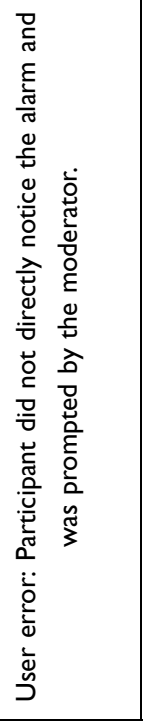 & & 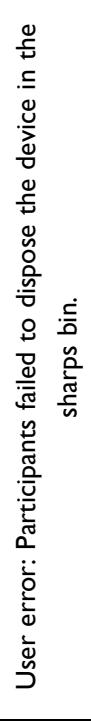 & & & 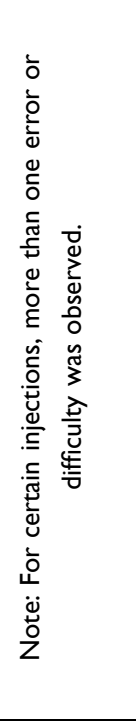 \\
\hline & 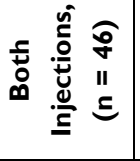 & 0 & $N$ & 0 & - & - & 0 & $\sigma$ & - & 0 & N & $=$ & $\underset{ }{\mathbb{O}}$ & 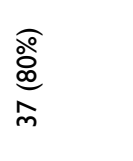 \\
\hline$\frac{1}{2}$ & 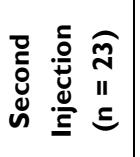 & 0 & $\sim$ & 0 & 0 & - & 0 & 0 & - & 0 & $N$ & 0 & ָั & $\begin{array}{l}\stackrel{\infty}{\infty} \\
\stackrel{\infty}{6} \\
\underline{\infty}\end{array}$ \\
\hline & 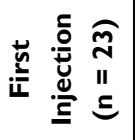 & 0 & 0 & 0 & - & 0 & 0 & $\sigma$ & 0 & 0 & 0 & in & స̃ & $\begin{array}{l}\stackrel{0}{\stackrel{\circ}{0}} \\
\stackrel{\infty}{\alpha} \\
\underline{a}\end{array}$ \\
\hline $\begin{array}{l}0 \\
\vdots \\
\vdots \\
n\end{array}$ & 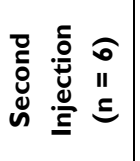 & & - & & & & & & & & & - & $\frac{n}{0}$ & 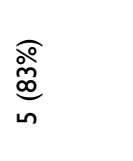 \\
\hline $\begin{array}{l}0 \\
j \\
j\end{array}$ & 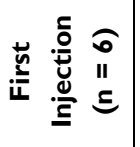 & & & & & & & - & & & & - & $\frac{1}{0}$ & 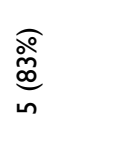 \\
\hline $\begin{array}{l}E \\
1 \\
0 \\
0\end{array}$ & 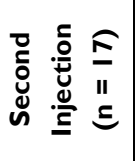 & & - & & & - & & & - & & $N$ & in & స్రి & $\begin{array}{l}\stackrel{\circ}{\circ} \\
\stackrel{0}{0} \\
\text { m}\end{array}$ \\
\hline 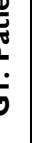 & 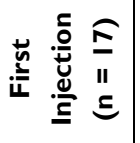 & & & & - & & & $m$ & & & & $\sigma$ & $\underset{ }{\stackrel{\Delta}{O}}$ & 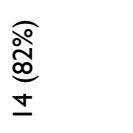 \\
\hline 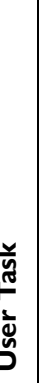 & & 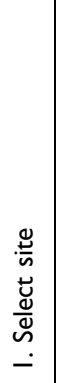 & 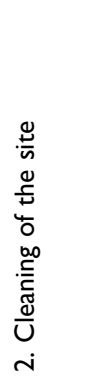 & 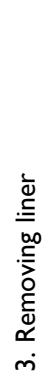 & 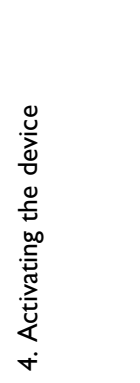 & 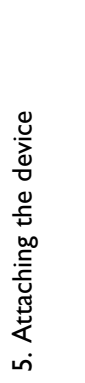 & 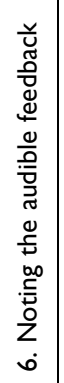 & 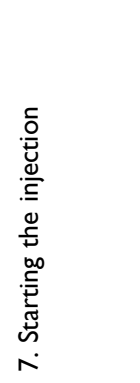 & 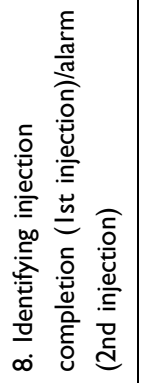 & 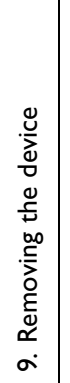 & 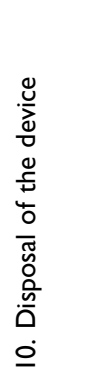 & 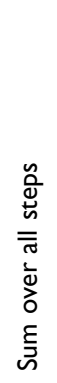 & 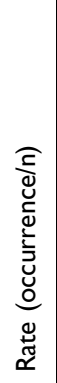 & 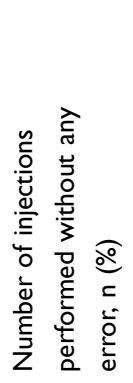 \\
\hline
\end{tabular}


U.1. I did not need to learn a lot of things before I could start using the device. (RC*)

U.2. I would imagine that most people would learn to use this device quickly.

U.3. I felt confident using this device.

U.4. It was easy to keep track of what step I was on with this device.

U.5. It was easy to hear the sounds that the device made.

U.6. It was easy to detect when the device had finished the injection.

U.7. The device was not cumbersome (or awkward) to use. (RC*)

U.8. It was easy to detect if the device had an error.

U.9. I did not feel nervous/ anxious when I detected the error. (RC*)

\begin{tabular}{|c|c|c|c|c|}
\hline Patients & $47 \%$ & $24 \%$ & $12 \%$ & $18 \%$ \\
\hline $\mathrm{HCP}$ & & & & $50 \%$ \\
\hline
\end{tabular}

\begin{tabular}{c|ccc|c|} 
Patients & $\mathbf{6 5 \%}$ & & $35 \%$ & \\
HCPs & $50 \%$ & $17 \%$ & $33 \%$
\end{tabular}
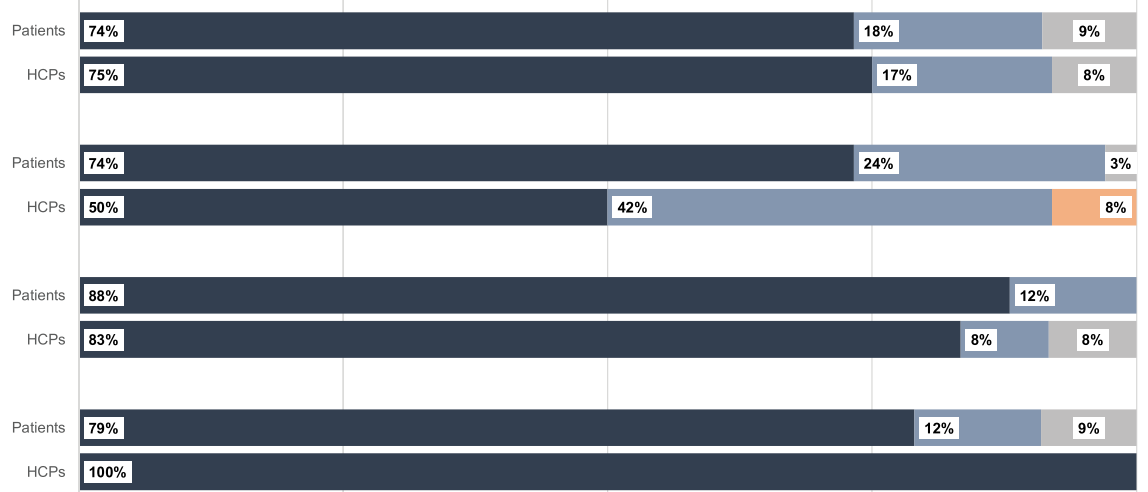

\begin{tabular}{|c|c|c|c|c|c|}
\hline Patients & $68 \%$ & & $3 \%$ & $15 \%$ & $15 \%$ \\
\hline $\mathrm{HCPs}$ & $55 \%$ & $18 \%$ & & $18 \%$ & $\mathbf{9} \%$ \\
\hline
\end{tabular}

\begin{tabular}{l|l|l}
\hline Patients & $88 \%$ & $12 \%$
\end{tabular}

$\mathrm{HCPs} 100 \%$

\begin{tabular}{|c|c|c|c|c|c|}
\hline Patients & $29 \%$ & $12 \%$ & $12 \%$ & $29 \%$ & $18 \%$ \\
\hline HCPs & $40 \%$ & & & $20 \%$ & $20 \%$ \\
\hline
\end{tabular}

Perceived usability based on participant group

Strongly agree

Agree

Neutral

Disagree

Strongly disagree

Figure 5 Self-reported ease of use of the device during the simulated injections.

Note: Items marked with RC* were reverse coded, ie, the questions were formulated as negative statements.

volume patch injector, with more than $70 \%$ of respondents "agreeing" or "strongly agreeing" with the different ease-of-use items (mean numerical scores were $>4$ on the five-point Likert scale). The rating was lower only for the item U.9., ie, "I did not feel nervous/ anxious when I detected the error," with $40 \%$ of patients (G1) and $60 \%$ of HCPs (G2) agreeing or strongly agreeing (mean scores of 3.05 and 3.40, respectively). Both patient and HCP participants rated similar values in terms of their confidence when using the device, feedback provided during injection, and error detection. The groups responded differently only for the two items related to learning (U.1. and U.2.), with HCPs expecting higher efforts to be required than patients.

\section{Wearability}

In general, patient participants were positively surprised by how easily the patch injector could be worn on the body during the simulated injections. With the exception of the first injection for the participant with excessive body hair, the device was secured effectively on the body throughout the study activities. Body size and shape was observed to vary significantly across the participants and could be expected to affect the wearability of a patch injector. In addition to body shape, other factors can be expected to influence successful placement of a patch injector, such as irritated skin, moles, stretch marks, and patches of body hair. The only issues related to wearability that were noted in the current study were the previously 
Table 4 Descriptive Statistics for Self-Reported Ease of Use of the Device During the Simulated Injections

\begin{tabular}{|c|c|c|c|c|c|}
\hline Item & User Group & Mean & Std. Dev. & Min & Max \\
\hline \multirow[t]{2}{*}{ U.I. I did not need to learn a lot of things before I could start using the device. (RC*) } & Patient $(n=17)$ & 4.00 & 1.17 & 2 & 5 \\
\hline & $\mathrm{HCP}(\mathrm{n}=6)$ & 3.50 & 1.64 & 2 & 5 \\
\hline \multirow[t]{2}{*}{ U.2. I would imagine that most people would learn to use this device quickly } & Patient $(n=17)$ & 4.65 & 0.49 & 4 & 5 \\
\hline & $\mathrm{HCP}(\mathrm{n}=6)$ & 4.17 & 0.98 & 3 & 5 \\
\hline \multirow[t]{2}{*}{ U.3. I felt confident using this device } & Patient $(n=34)$ & 4.65 & 0.65 & 3 & 5 \\
\hline & $\mathrm{HCP}(\mathrm{n}=12)$ & 4.67 & 0.65 & 3 & 5 \\
\hline \multirow[t]{2}{*}{ U.4. It was easy to keep track of what step I was on with this device } & Patient $(n=34)$ & 4.71 & 0.52 & 3 & 5 \\
\hline & $\mathrm{HCP}(\mathrm{n}=12)$ & 4.33 & 0.89 & 2 & 5 \\
\hline \multirow[t]{2}{*}{ U.5. It was easy to hear the sounds that the device made } & Patient $(n=34)$ & 4.82 & 0.46 & 3 & 5 \\
\hline & $\mathrm{HCP}(\mathrm{n}=12)$ & 4.75 & 0.62 & 3 & 5 \\
\hline \multirow[t]{2}{*}{ U.6. It was easy to detect when the device had finished the injection } & Patient $(n=34)$ & 4.71 & 0.63 & 3 & 5 \\
\hline & $\mathrm{HCP}(\mathrm{n}=1 \mathrm{I})$ & 5.00 & 0 & 5 & 5 \\
\hline \multirow[t]{2}{*}{ U.7. The device was not cumbersome (or awkward) to use. (RC*) } & Patient $(n=34)$ & 4.24 & 1.18 & 2 & 5 \\
\hline & $\mathrm{HCP}(\mathrm{n}=1 \mathrm{I})$ & 4.18 & 1.08 & 2 & 5 \\
\hline \multirow[t]{2}{*}{ U.8. It was easy to detect if the device had an error } & Patient $(n=17)$ & 4.88 & 0.33 & 4 & 5 \\
\hline & $\mathrm{HCP}(\mathrm{n}=5)$ & 5.00 & 0 & 5 & 5 \\
\hline \multirow[t]{2}{*}{ U.9. I did not feel nervous/ anxious when I detected the error. (RC*) } & Patient $(n=17)$ & 3.06 & 1.56 & I & 5 \\
\hline & $\mathrm{HCP}(\mathrm{n}=5)$ & 3.40 & 1.82 & I & 5 \\
\hline
\end{tabular}

Note: Items marked with RC* were reverse coded, ie, the questions were formulated as negative statements.

mentioned cases of excessive body hair and small body size.

For patient participants, the comfort and security of the patch injector were tested through multiple movement-based tasks during the simulated injection, with success almost universal across participants. For both simulated injections, participants were tasked with predefined movement-based tasks, including reaching up, bending down to "tie their shoe," and walking around in a circle. In addition, participants were asked to complete a task of packing or unpacking groceries, which involved lifting and bending movements, which also distracted them from the device. Across the tasks that the participants performed, there were only a few instances where the device interfered with their movement, and none in a critical manner such that the injection could have been interrupted. Certain participants were surprised by this, which suggests that the initial perception of the largevolume patch injector differed from the actual user experience. Additionally, features of the simulated use environment such as a refrigerator door did not obstruct or interfere with the functioning of the device.

Moreover, participants indicated that the patch injector was easily forgotten during the tasks. Wearers quickly reached the point of sensory satiety, more when the device was attached to the abdomen than the thigh. This is a positive attribute as the device does not seem to call attention to itself, neither because of its size nor because of a potential pull from the adhesive while moving.

After completing the movement tasks, patient participants completed a questionnaire providing feedback on the perceived wearability of the device. The results are presented in Figure 6 and Table 5, and indicate that users rated the wearability highly, with approximately $80 \%$ of respondents either "agreeing" or "strongly agreeing" with the proposed items in terms of wearing comfort (mean numerical scores were considerably $>4$ on the five-point Likert scale). Thus, despite perceiving the device to be large, participants expressed that the patch injector was comfortable when worn. Here, it may be noted that the ratings for abdomen and thigh placement in terms of comfort were almost identical, whereas removal was rated to be somewhat easier when the device was worn on the thigh. The participant ratings on perceived security while wearing the device were marginally lower than the comfort ratings, with participants viewing the abdomen as a more secure spot for the patch injector than the thigh. 


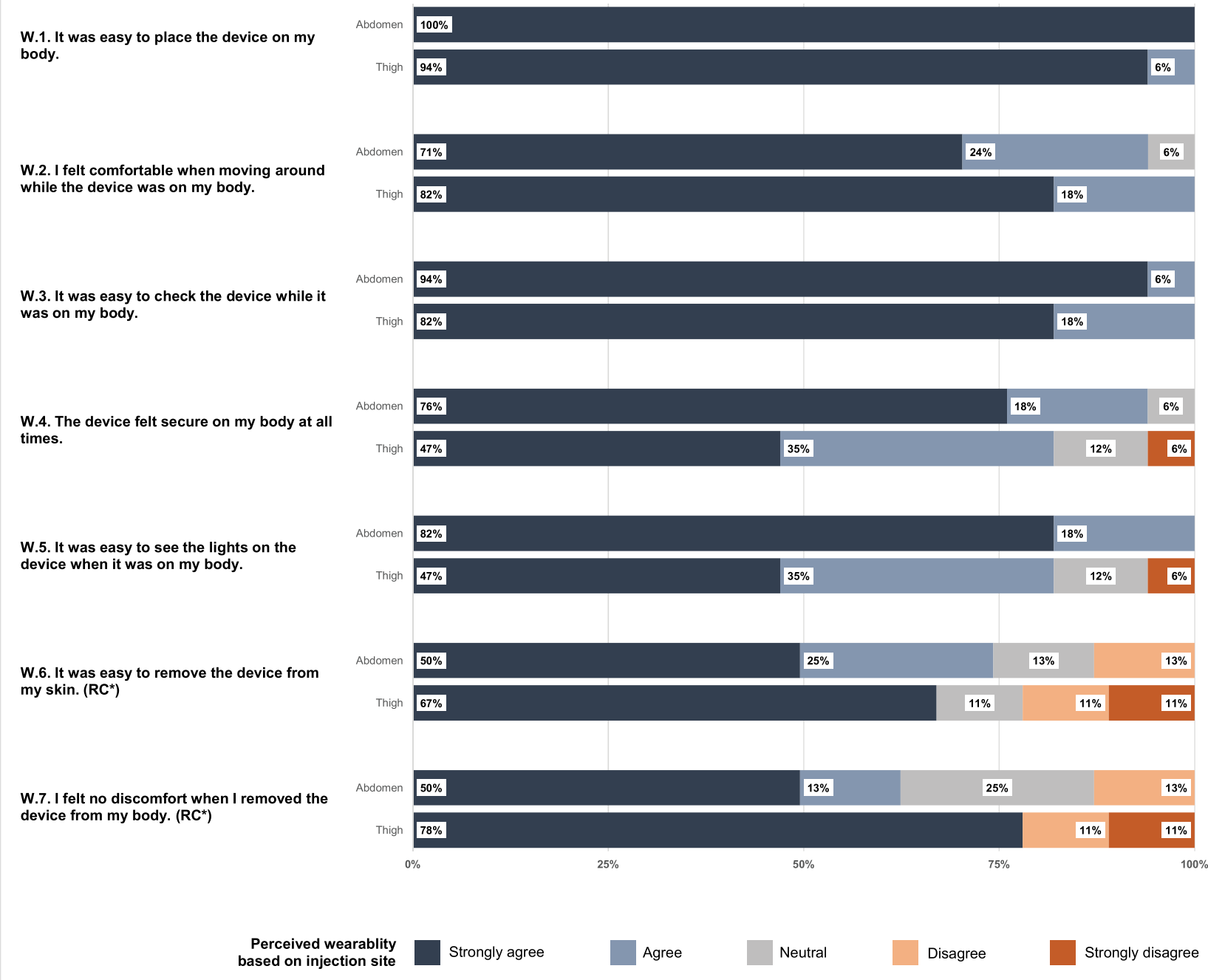

Figure 6 Self-reported wearability of the device during the simulated injections.

Note: Items marked with RC* were reverse coded, ie, the questions were formulated as negative statements.

\section{Discussion}

This study investigated the usability (ie, overall handling) and wearability (ie, acceptability of size and weight) through simulated injections using a large-volume patch injector device intended to deliver single doses up to $10 \mathrm{~mL}$. The primary findings were that although participants generally had a positive first impression of the device and injection technology, they were initially rather skeptical about the size of the device. In terms of usability, participants were predominantly successful in performing the simulated injections with few user errors or difficulties. The self-reported rating of usability was high. In terms of the wearability of the patch injector, all participants were able to place the device on their bodies, irrespective of body size or habitus, and found the injector comfortable to wear. Participants were able to move around and perform predefined activity tasks with the device attached to them and rated the different wearability aspects positively.

The only significant usability issue observed in the study was that four participants faced difficulties in activating the patch injector after having attached it to their bodies. A root cause analysis determined that the difficulties were caused by confusing signals and feedback from the device at this point during use. The issue was subsequently addressed by changes to the pattern of visual and acoustical feedback provided by the patch injector during activation, attachment, and initialization of the injection process. 
Table 5 Descriptive Statistics for Self-Reported Wearability of the Device During the Simulated Injections

\begin{tabular}{|c|c|c|c|c|c|}
\hline Variable & Placement & Mean & Std. Dev. & Min & Max \\
\hline \multirow[t]{2}{*}{ W.I. It was easy to place the device on my body } & Abdomen $(n=17)$ & 5.00 & 0 & 5 & 5 \\
\hline & Thigh $(n=17)$ & 4.94 & 0.24 & 4 & 5 \\
\hline \multirow[t]{2}{*}{ W.2. I felt comfortable when moving around while the device was on my body } & Abdomen $(n=17)$ & 4.65 & 0.61 & 3 & 5 \\
\hline & Thigh $(n=17)$ & 4.82 & 0.39 & 4 & 5 \\
\hline \multirow[t]{2}{*}{ W.3. It was easy to check the device while it was on my body } & Abdomen $(n=17)$ & 4.95 & 0.24 & 4 & 5 \\
\hline & Thigh (17) & 4.82 & 0.39 & 4 & 5 \\
\hline \multirow[t]{2}{*}{ W.4. The device felt secure on my body at all times } & Abdomen $(n=17)$ & 4.71 & 0.59 & 3 & 5 \\
\hline & Thigh $(n=17)$ & 4.18 & 1.07 & I & 5 \\
\hline \multirow[t]{2}{*}{ W.5. It was easy to see the lights on the device when it was on my body } & Abdomen $(n=17)$ & 4.82 & 0.39 & 4 & 5 \\
\hline & Thigh $(n=17)$ & 4.76 & 0.75 & 2 & 5 \\
\hline \multirow[t]{2}{*}{ W.6. It was easy to remove the device from my skin. (RC*) } & Abdomen $(n=8)$ & 4.13 & 1.13 & 2 & 5 \\
\hline & Thigh $(n=9)$ & 4.00 & 1.58 & I & 5 \\
\hline \multirow[t]{2}{*}{ W.7. I felt no discomfort when I removed the device from my body. (RC*) } & Abdomen $(n=8)$ & 4.00 & 1.20 & 2 & 5 \\
\hline & Thigh $(n=9)$ & 4.22 & 1.56 & I & 5 \\
\hline
\end{tabular}

Note: Items marked with RC* were reverse coded, ie, the questions were formulated as negative statements.

In the current study, use error rates of 0.26 errors per injection for the first injection and 0.22 errors per injection for the second injection were observed. These results are in good agreement with prior empirical studies on largevolume wearable devices using different user groups. A recent work on a wearable infusion pump using patients with pulmonary arterial hypertension reported a similar error rate of 0.23 errors per injection (14 errors observed for 60 injections; no distinction was made between first and second injections), which indicates a similar handling complexity between the two devices under investigation. ${ }^{31}$ The error rates reported here are also consistent with, or even lower than, those reported for injection pens and autoinjectors, both of which are widely used for subcutaneous injections and the latter is considered as an alternative to large-volume patch injectors for various drugs. ${ }^{6,32-34}$ For instance, error rates of 2.39 errors per injection for a first injection and 1.94 errors for a second injection were reported for variable doses using an insulintype pen while considering a similar broad user population. ${ }^{35}$ For fixed-dose pen injectors with a single preset dose, 0.70 and 0.65 errors per injection for the first and second injections, respectively, were observed. ${ }^{36} \mathrm{An}$ autoinjector study based on patient population across chronic disease states measured 0.21 and 0.33 errors per injection for the first and second simulated use, respectively. ${ }^{37}$ Moreover, a recent summative usability testing of a novel prefilled autoinjector based on type 2 diabetes patients only showed 0.22 errors per injection (only first injection performed). ${ }^{38}$ The error rates observed in the current large-volume patch injector study are lower than those observed for pen injectors and are highly comparable to rates observed for autoinjector devices. This is because prefilled large-volume patch and autoinjector-type devices are less complicated to use than pens, which require the user to attach a separate pen needle, perform a function test, and, in the case of insulin-type pens, dial the desired dose prior to injecting the target dose. Moreover, the small learning effect (understood as the change in error rate between the first and second injections) that was observed in the current study in the case of patient users of the large-volume patch injector is similar to the learning effects reported for the different pen injectors.

In terms of self-reported usability, HCPs provided lower ratings than patients, although all ratings were high and the differences between groups were small. Understanding the perspectives of HCPs is particularly important as they are likely to continue to be involved in subcutaneous drug administration using novel largevolume patch devices in oncology, where the development of subcutaneous formulations has recently attracted significant interest. ${ }^{39-42}$ Although other studies have reported similar differences in rating between professional and lay 
users, ${ }^{35}$ the reason for the skepticism of HCPs toward such devices remains poorly understood. Future research may aid in overcoming the skepticism of HCPs, which will aid in the commercialization of this type of device.

The positive results reported in the current study in terms of wearability compare well with the only other known published study on a similar type of large-volume patch injector. ${ }^{22}$ This is noteworthy considering the initial reaction of participants to the size of the device, the fact that the device holds up to $10 \mathrm{~mL}$, and that injections last up to $20 \mathrm{~min}$. The previous study reported similar positive self-evaluations; however, the device in that experiment was $25 \%$ smaller in size $(5 \mathrm{~mL}$ fill volume only) and had a $67 \%$ shorter wearing time than the device used in the current study. ${ }^{22}$

The choice of the injection site (abdomen or thigh) had a limited effect on perceived wearability. Patients rated most aspects of perceived wearability the same regardless of the injection site, with the exception of perceived security of attachment, which was rated higher for the abdomen as the injection site, and ease of removal, which was rated higher for the thigh as the injection site. These differences may be related to the size of the device that can hold a $10 \mathrm{~mL}$ cartridge. The study with the smaller $5 \mathrm{~mL}$ device did not show any differences in preference between placement on the abdomen or thigh. $^{22}$

These insights into the usability and wearability of the large-volume injector inform the further path to clinical use in two ways. First, the results form the basis for final late-stage design refinements of the device platform to enable safe and effective self-administration of therapeutics for chronic diseases. Second, the study also facilitates subsequent customization of the platform into drugspecific device derivatives that will enter clinical use by identifying the needs of specific user groups. The results of the current study should also be interpreted considering its limitations, in particular, the simulated nature of the study, small number of participants, and the fact that it was not conducted in a home setting. Particularly, the absence of a needle in the prototype devices may well have influenced participants' perception of wearability when performing movement activities.

\section{Conclusion}

The participants of the simulated use study successfully used a novel high-volume patch injector regardless of disease state, age, or body size and habitus. Patients and HCPs were generally successful in performing the simulated injections, and only a few errors or difficulties were observed; moreover, the perceived usability was rated positively. All participants were able to place the device on their bodies, irrespective of body size or habitus, and found the injector comfortable to wear. Patient participants were able to move around and perform tasks with the attached device and rated the different wearability aspects positively. These results indicate that the large-volume patch injector reported here can be safely and effectively used by the intended broad patient and HCP population.

\section{Ethics Approval and Informed Consent}

The study complied with the principles of the declaration of Helsinki. The study protocol, informed consent form, and relevant supporting information were reviewed and approved by an institutional review board, Advarra IRB (Pro00037054), prior to study initiation. Participants, and parents or legal guardians for participants under the age of 18 , provided informed consent, and also consented to the publication of anonymized responses from the study.

\section{Acknowledgments}

The authors would like to thank Philippe Mueller for his support in conducting the descriptive statistical analysis.

\section{Funding}

This study was financed by Ypsomed AG and conducted by Insight Product Development.

\section{Disclosure}

JL, AS, and CJ work for Ypsomed AG. ML and TD work for Insight Product Development. The authors have no other relevant affiliations or financial involvement to declare.

\section{References}

1. Jones GB, Collins DS, Harrison MW, Thyagarajapuram NR, Wright JM. Subcutaneous drug delivery: an evolving enterprise. Sci Transl Med. 2017;9(405):405. doi:10.1126/scitranslmed.aaf9166

2. Viola M, Sequeira J, Seiça R, et al. Subcutaneous delivery of monoclonal antibodies: how do we get there? $J$ Control Release. 2018;286:301-314.

3. Pivot X, Gligorov J, Müller V, et al. Patients' preferences for subcutaneous trastuzumab versus conventional intravenous infusion for the adjuvant treatment of HER2-positive early breast cancer: final analysis of 488 patients in the international, randomized, two-cohort PrefHer study. Ann Oncol. 2014;25(10):1979-1987.

4. Rummel M, Kim T, Aversa F, et al. Preference for subcutaneous or intravenous administration of rituximab among patients with untreated CD20+ diffuse large B-cell lymphoma or follicular lymphoma: results from a prospective, randomized, open-label, crossover study (PrefMab). Ann Oncol. 2017;28(4):836-842. 
5. Bittner B, Richter W, Schmidt J. Subcutaneous administration of biotherapeutics: an overview of current challenges and opportunities. BIoDrugs. 2018;32(5):425-440.

6. Kivitz A, Baret-Cormel L, van Hoogstraten H, et al. Usability and patient preference Phase 3 study of the sarilumab pen in patients with active moderate-to-severe rheumatoid arthritis. Rheumatol Ther. 2018;5(1):231-242.

7. Schiff M, Koo J, Jin E, et al. Usability and acceptability of the abatacept pre-filled autoinjector for the subcutaneous treatment of rheumatoid arthritis. Adv Ther. 2016;33(2):199-213.

8. Callis Duffin K, Bukhalo M, Bobonich MA, et al. Usability of a novel disposable autoinjector device for ixekizumab: results from a qualitative study and an open-label clinical trial, including patient-reported experience. Med Devices (Auckl). 2016;9:361-369.

9. Ziemssen T, Sylvester L, Rametta M, Ross AP. Patient satisfaction with the new interferon Beta-1b autoinjector (BETACONNECT). Neurol Ther. 2015;4(2):125-136.

10. Limmroth V, Gerbershagen K. Single-use autoinjector for onceweekly intramuscular injection of IFN $\beta-1$ a. Expert Opinion Drug Delivery. 2014;11(12):1969-1978. doi:10.1517/17425247.2014. 943181

11. Bruin G, Hockey H-UP, La Stella P, et al. Comparison of pharmacokinetics, safety and tolerability of secukinumab administered subcutaneously using different delivery systems in healthy volunteers and in psoriasis patients. Br J Clin Pharmacol. 2020;86(2):338-351. doi:10.1111/bcp.14155

12. Portron A, Jordan P, Draper K, et al. A Phase I study to assess the effect of speed of injection on pain, tolerability, and pharmacokinetics after high-volume subcutaneous administration of gantenerumab in healthy volunteers. Clin Ther. 2020;42(1):108-120. e101.

13. Schneider A, Mueller P, Jordi C, et al. Hold the device against the skin: the impact of injection duration on user's force for handheld autoinjectors. Expert Opin Drug Deliv. 2020;17(2):225-236.

14. Mathaes R, Koulov A, Joerg S, Mahler H-C. Subcutaneous injection volume of biopharmaceuticals - pushing the boundaries. J Phar Sci. 2016;105(8):2255-2259.

15. Connor RJ, Taverna DM, Thrall K, LaBarre MJ, Kang DW. Use of computed tomography to assess subcutaneous drug dispersion with recombinant human hyaluronidase $\mathrm{PH} 20$ in a swine model. J Pharmacol Toxicol Methods. 2020;106:106936.

16. Stewart KD, Johnston JA, Matza LS, et al. Preference for pharmaceutical formulation and treatment process attributes. Patient Prefer Adherence. 2016;10:1385-1399.

17. Collins D, Sánchez-Félix M, Badkar AV, Mrsny R. Accelerating the development of novel technologies and tools for the subcutaneous delivery of biotherapeutics. $J$ Control Release. 2020;321:475-482.

18. Joshi RS, Egbuna OI, Cairns AS, et al. Performance of the pegfilgrastim on-body injector as studied with placebo buffer in healthy volunteers. Curr Med Res Opin. 2017;33(2):379-384.

19. Ginsberg BH. Patch Pumps for Insulin. J Diabetes Sci Technol. 2019;13(1):27-33.

20. Wiggins BS, Senfield J, Kassahun H, Lira A, Somaratne R. Evolocumab: considerations for the management of hyperlipidemia. Curr Atheroscler Rep. 2018;20(4):1-13.

21. Badkar AV, Gandhi RB, Davis SP, LaBarre MJ. Subcutaneous delivery of high-dose/volume biologics: current status and prospect for future advancements. Drug Des Devel Ther. 2021;15:159-170.

22. Woodley WD, Yue W, Morel DR, Lainesse A, Pettis RJ, Bolick NG. Clinical evaluation of an investigational $5 \mathrm{~mL}$ wearable injector in healthy human subjects. Clin Transl Sci. 2021;14(3):859.

23. Dias C, Abosaleem B, Crispino C, Gao B, Shaywitz A. Tolerability of high-volume subcutaneous injections of a viscous placebo buffer: a randomized, crossover study in healthy subjects. AAPS PharmSciTech. 2015;16(5):1101-1107.
24. Berteau C, Filipe-Santos O, Wang T, Rojas HE, Granger C, Schwarzenbach F. Evaluation of the impact of viscosity, injection volume, and injection flow rate on subcutaneous injection tolerance. Med Devices (Auckl). 2015;8:473-484.

25. Zijlstra E, Jahnke J, Fischer A, Kapitza C, Forst T. Impact of injection speed, volume, and site on pain sensation. $J$ Diabetes Sci Technol. 2018;12(1):163-168.

26. Allmendinger A, Mueller R, Schwarb E, et al. Measuring tissue backpressure-in vivo injection forces during subcutaneous injection. Pharm Res. 2015;32(7):2229-2240.

27. Heise T, Nosek L, Dellweg S, et al. Impact of injection speed and volume on perceived pain during subcutaneous injections into the abdomen and thigh: a single-centre, randomized controlled trial. Diabetes Obes Metab. 2014;16(10):971-976.

28. Jain M, Doughty D, Clawson C, et al. Tralokinumab pharmacokinetics and tolerability when administered by different subcutaneous injection methods and rates. Int J Clin Pharmacol Ther. 2017;55 (7):606-620.

29. Association UP. UPA Code of Professional Conduct for Usability Practitioners. Usability Professionals' Association; 2005.

30. Association EPMR. EphMRA 2017 Code of Conduct V4. European Pharmaceutical Market Research Association; 2017.

31. Noymer P, Shaked A, Newell K. Human factors and usability engineering in the development of SMT-101 for the treatment of pulmonary arterial hypertension. Expert Opin Drug Deliv. 2018;15 (4):325-333.

32. LaRue S, Springer J, Noderer M, Meehan J, Wysham CH. Evaluation of the use of exenatide once-weekly suspension autoinjector among patients with Type 2 diabetes mellitus and health care professionals. J Diabetes Sci Technol. 2019;13(2):226-234.

33. Vermeire S, D'Heygere F, Nakad A, et al. Preference for a prefilled syringe or an auto-injection device for delivering golimumab in patients with moderate-to-severe ulcerative colitis: a randomized crossover study. Patient Prefer Adherence. 2018;12:1193-1202.

34. Stauffer VL, Sides R, Lanteri-Minet M, et al. Comparison between prefilled syringe and autoinjector devices on patient-reported experiences and pharmacokinetics in galcanezumab studies. Patient Prefer Adherence. 2018;12:1785-1795.

35. Lange J, Richard P, Bradley N. Usability of devices for self-injection: results of a formative study on a new disposable pen injector. Med Devices (Auckl). 2014;7:195-203.

36. Lange J, Nemeth T. Formative usability evaluation of a fixed-dose pen-injector platform device. Med Devices (Auckl). 2018;11:105-112.

37. Lange J, Richard P, Bradley N. Usability of a new disposable autoinjector platform device: results of a formative study conducted with a broad user population. Med Devices (Auckl). 2015;8:255-264.

38. Klonoff DC, Bassock S, Dwyer A, et al. Evaluating the usability and safety of the semaglutide single-dose pen-injectors through summative (human factors) usability testing. J Diabetes Investig. 2021;12 (6):978-987.

39. Pivot X, Gligorov J, Müller V, et al. Preference for subcutaneous or intravenous administration of trastuzumab in patients with HER2-positive early breast cancer (PrefHer): an open-label randomised study. Lancet Oncol. 2013;14(10):962-970.

40. Usmani SZ, Nahi H, Mateos M-V, et al. Subcutaneous delivery of daratumumab in relapsed or refractory multiple myeloma. Blood. 2019;134(8):668-677.

41. Johnson ML, Braiteh F, Grilley-Olson JE, et al. Assessment of subcutaneous vs intravenous administration of anti-PD-1 antibody PF-06801591 in patients with advanced solid tumors: a Phase 1 dose-escalation trial. JAMA Oncol. 2019;5(7):999-1007.

42. Merz M, Salwender H, Haenel M, et al. Subcutaneous versus intravenous bortezomib in two different induction therapies for newly diagnosed multiple myeloma: an interim analysis from the prospective GMMG-MM5 trial. Haematologica. 2015;100(7):964-969. 


\section{Publish your work in this journal}

Medical Devices: Evidence and Research is an international, peerreviewed, open access journal that focuses on the evidence, technology, research, and expert opinion supporting the use and application of medical devices in the diagnosis, monitoring, treatment and management of clinical conditions and physiological processes. The identification of novel devices and optimal use of existing devices which will lead to improved clinical outcomes and more effective patient management and safety is a key feature of the journal. The manuscript management system is completely online and includes a very quick and fair peer-review system. Visit http:// www.dovepress.com/testimonials.php to read real quotes from published authors. 